\title{
Histopathological Changes of the Retina After Nd: YAG Laser Thrombolysis in Branch Retinal Vein Occlusion: An Experimental Study
}

\author{
Salwa Abdelkawi Ahmed', Aziza Ahmed Hassan², Dina Fouad Ghoneim², Ahmed Tamer Sayed Saif ${ }^{3}$ \\ 'Department of Vision Science, Biophysics and Laser Science Unit, Research Institute of Ophthalmology, Giza, Egypt \\ ${ }^{2}$ Ophthalmic Unit, National Institute for Laser Enhanced Science, Cairo University, Egypt \\ ${ }^{3}$ Ophthalmic Unit, Fayoum university, Egypt
}

\author{
*Correspondence to \\ Salwa Abdelkawi Ahmed, Vision \\ Sciences Department, Biophysics \\ and Laser Science Unit, Research \\ Institute of Ophthalmology, 2 El- \\ ahram St. Giza, Egypt. Postal code: \\ 12511. \\ Fax: +20235735688 \\ Tel: +20100 1670590; \\ Email: saelkawi@yahoo.com
}

Published online 15 December 2018

\begin{abstract}
Introduction: The efficacy of many therapeutics techniques for treatment of branch retinal vein occlusion (BRVO) has been the subject of many investigations. The aim of the present work is to evaluate the transluminal $\mathrm{Nd}$ : YAG laser thrombolysis as a new therapeutic approach used for treatment of BRVO in rabbits as an experimental model.

Methods: Four rabbits were considered as a control ( $n=8$ eyes); occlusion of the branch retinal veins was performed by using a dye enhancing thrombus formation in right eyes of 10 rabbits $(n=10$ eyes). Thrombi in the retinal veins were induced by intravenous injection of rose bengal solution as a photosensitizer immediately before the argon laser application with a power of $1200 \mathrm{~mW}$, a spot size of $100 \mu \mathrm{m}$, and a duration of $20 \mathrm{~ms}$. One week later, transluminal Nd: YAG laser thrombolysis ( $30 \mathrm{~mJ}, 3$ pulses/4 ns) was employed to the site of occluded veins, until the thrombi were partially or completely shattered. The rabbits were followed up after 4 days, 1 week and 2 weeks for slit lamp fundus examination and the treated retinas were isolated for histopathological examination.

Results: Argon laser photothrombosis induced complete BRVO with some vitreous hemorrhage, destruction, and necrosis in the surrounding retinal layers. Moreover, one week later, Nd: YAG laser thrombolysis showed complete venous flow, minimal vitreous hemorrhage, reperfused retina, complete veins improvement. Follow up after 2 weeks revealed more improvement of all retinal layers.

Conclusion: Treatment with transluminal Nd: YAG laser thrombolysis represented a novel therapeutic modality in BRVO.

Keywords: Branch retinal vein occlusion; Photothrombosis; Rose bengal; Thrombolysis; Lasers.
\end{abstract}

\section{Introduction}

Retinal vascular occlusive disease was considered as one of the major causes of blindness and there is marked argument on its clinical features, management and its pathogenesis. ${ }^{1,2}$ Retinal vein occlusion (RVO) is the commonest sight threatening vascular disease after diabetic retinopathy. ${ }^{3} \mathrm{RVO}$ is an obstruction in the retinal veins due to thrombus formation. It was firstly described clinically as retinal apoplexy. ${ }^{4}$ In addition, it occurs equally in both sexes especially in middle age and older people with systemic arterial hypertension, diabetes mellitus and atherosclerotic disease. ${ }^{5}$ Early treatment and recognition of RVO are important to avoid significant visual disease. ${ }^{6}$ So that research on prevention and treatment approaches for this sight threatening eye disease is needed. ${ }^{2}$
It is well known that, there are three basic types of vein occlusion namely, central retinal vein occlusion (CRVO); branch retinal vein occlusion (BRVO); and hemi-CRVO. ${ }^{2}$ CRVO was divided into ischemic and nonischemic types while, BRVO is more common than CRVO and was divided into major BRVO and macular BRVO. Moreover, macular edema was present in $30 \%$ of BRVO cases. ${ }^{7}$ Hemi-CRVO is occurred in only one half of the retina surface and is divided into ischemic and nonischemic types. $^{6}$

Compression of the retinal veins by the relatively rigid artery may result in endothelial damage, turbulent flow, thrombosis and occlusion. ${ }^{8}$ Many local anatomic, systemic, ocular and thrombogenic risk factors are associated with BRVO. ${ }^{9}$ The anatomic risk factor includes arteriovenous crossing, and systemic risk factors include

Please cite this article as follows: Abdelkawi SA, Hassan AA, Ghoneim DF, Sayed Saif AT. Histopathological changes of the retina after Nd: YAG laser thrombolysis in branch retinal vein occlusion: an experimental study. J Lasers Med Sci. 2019;10(1):50-55. doi:10.15171/ jlms.2019.08. 
cardiovascular disease, hypertension, diabetes mellitus, hyperlipidemia, increased body mass index at age 20 years, glaucoma, high levels of alpha 2-globulin and hematological disorders. ${ }^{10}$ In addition, ocular risk factors involve glaucoma and hyperopia. ${ }^{11}$ Furthermore, arterial hypertension and hyperviscosity are presented in the thrombogenic factors. ${ }^{12}$

Metabolic and systemic vascular diseases are very strongly connected with BRVO. ${ }^{13}$ Blood abnormalities play important roles in the pathogenesis of BRVO. Consequently, erythrocyte volume; level of fibrinogen; and hematocrit appeared to be substantial. ${ }^{14}$ Recent meta-analyses of RVO and thrombophilic factors showed that, hyperhomocysteinemia and anticardiolipin antibodies play a role in the pathogenesis of RVO. ${ }^{15-20}$

The main pathogenic mechanism for development of BRVO is arterial toughness that gives rise to venous compression in the common adventitial sheath.,21-24 According to the extent of macular damage, patients with BRVO may suffer from decreased vision acuity or vision loss. In the acute stage, the retina shows intraretinal hemorrhages, tortuosity and venous dilation, delayed venous filling, macular edema, and microvascular abnormalities. Recently, subretinal hemorrhage and serous macular detachment have been documented by using optical coherence tomography. ${ }^{25}$

RVO pathogenesis is still not completely comprehended due to a combination of three systemic changes: degenerative changes of the vessel wall (1), hemodynamic changes (venous stasis) (2), and blood hypercoagulability (3). ${ }^{15}$ Many therapies have been evaluated in the treatment of RVO such as laser intravitreal steroids, photocoagulation, anti-vascular endothelial growth factor (anti-VEGF) agents, hemodilution, pars plana vitrectomy, anticoagulation therapy and fibrinolysis. Due to its multifactorial nature, and no contributing treatments have been shown in large randomized studies seemed to be effective, so treatment of RVO is still a challenge. ${ }^{15}$ Thus, this study aimed to use transluminal Nd- YAG laser as a new treatment modality for experimental BRVO that induced in the rabbits' retina by rose bengal photodynamic therapy.

\section{Methods}

\section{Animals}

Fourteen male New Zealand rabbits were obtained from the animal house of Research Institute of Ophthalmology, Giza, Egypt. The animals were kept in a standard 12 hours of light and 12 hours of darkness cycle with free access to water and balanced diet. Right eyes of ten rabbits (weighing 3-3.5 $\mathrm{kg}$ ) were used ( $\mathrm{n}=10$ eyes) for creation of BRVO and eyes of four rabbits were served as a control. The rabbits were generally anesthetized using intramuscular Xyla-Ject $(0.2 \mathrm{~mL} / \mathrm{kg})$ followed by ketamine hydrochloride $(0.6 \mathrm{~mL} / \mathrm{kg})$ and benoxinate eye drops (0.4\%) was used for local anesthesia. Animals pupils were dilated with $1 \%$ cyclopentolate eye drops (Bausch \& Lomb U.K Limited). All procedures were directed in accordance to the principles articulated in the Guide for Care and Use of Laboratory Animals. They were subjected to experimental protocols agreed by the local experimental ethics committee of ophthalmic and vision research.

\section{Photodynamic Therapy Induced Vein Occlusion}

Occlusion of the branch retinal veins was performed by photodynamic therapy through dye enhanced photothrombosis. Thrombi were induced photochemically in the retinal veins using the method described previously by Oncel et al. ${ }^{26}$ Rose bengal (4,5,6,7-tetrachloro-2, $4^{\prime}, 5^{\prime}, 7^{\prime}$ tetraiodofluorescein) was purchased from Sigma-Aldrich Switzerland, Buchs, Switzerland. Fifty milligrams of rose bengal $/ \mathrm{ml}$ saline was injected in the middle ear vein immediately before argon laser photodynamic therapy application. Argon green laser (Vitra, France, $532 \mathrm{~nm}$ ) application with a power of $1.2 \mathrm{~W}$, a spot size of $100 \mu \mathrm{m}$, and duration of 20 milliseconds was performed within 5 minutes while the rose bengal was in the venous circulation. Each vessel was treated at a half to one disc diameter from the optic disc. About 5-20 laser shoots were applied for each vessel until the blood flow was completely stopped in the vein. Occlusion was confirmed when whitening of the blood vessel and stasis were seen by fundus observation using slit lamb biomicroscopic examination. The rabbits were injected with intramuscular alpha chymotrypsin day after day for one week to reduce inflammation and eye tissue destruction.

\section{Laser Thrombolysis}

After 1 week of rose bengal photodynamic therapy, transluminal Nd: YAG laser (Optimis II, France, 1064 $\mathrm{nm}$ ) thrombolysis was applied to the site of occluded veins. A Goldman 3 mirror lens was used to focus the Nd: YAG laser into the venous thrombus. The laser energy level was operated at $30 \mathrm{~mJ}$, about 12-15 pulses, spot size $10 \mu \mathrm{m}$, and duration of 3 pulses $/ 4.0 \mathrm{~ns}$ until the thrombus was completely removed.

Fundus and Histopathological Examination of the Retina One week after occlusion of branch retinal veins, fundus examination was performed and 4 rabbits were decapitated ( $\mathrm{n}=4$ eyes) for histopathological examination. In addition, thrombolysis that occurred after application of transluminal Nd: YAG laser was confirmed in the rest of rabbits ( $n=6$ eyes) by slit lamb biomicroscopic funds examination. At the end of the estimated periods, the rest of the rabbits were sacrificed after 1 and 2 weeks (three rabbits each). The eyes were instantly enucleated, and fixed in $2.5 \%$ glutaraldehyde in phosphate buffer for half an hour and then the retinae were carefully removed from the posterior chamber of the eye for histopathological 
examination. The retinae specimens were dissected into small pieces and instantly fixed in $2.5 \%$ phosphate buffered glutaraldehyde ( $\mathrm{pH} 7.4$ ) at $4^{\circ} \mathrm{C}$ for 6 hours. The specimens were fixed in $1 \%$ osmium tetra-oxide for 30 minutes, dehydrated in an ascending grade of ethanol and then embedded in araldite CY212 mixtures. Semithin sections were cut $(1 \mu \mathrm{m})$ and stained with toluidine blue for light microscope examination.

\section{Results}

Fundus Examination

The slit lamp biomicroscopic fundus examination for control rabbits showed normal retina and vascular complex (Figure 1A). Argon laser was applied after injection of intravenous rose bengal to induce BRVOs in ten rabbit's eyes (Figure $1 \mathrm{~B}$ and $1 \mathrm{C}$ ). Following the photothrombosis, the area of irradiated retinal vein was narrow with complete BRVOs. Four days later, fundus examination revealed vitreous hemorrhage, venous tortuosity, blurred disc edge and hemorrhage around disc (Figure 1D). One week after photothrombosis, slit lamp biomicroscopic fundus examination showed disappearance of vitreous hemorrhage (Figure 1C). BRVO in the right eye of all rabbits were treated by $\mathrm{Nd}$ : YAG laser till thrombolysis occurred and flow of blood stream was attained (Figure $2 \mathrm{~A})$. In some rabbits, injury to venous wall and stream of blood flow occurred with improvement of the pale colored retina (Figure 2B). Follow up after one week using slit lamp biomicroscopic fundus examination revealed complete venous flow, minimal vitreous hemorrhage, and dry pale retina (Figure 2C). After 2 weeks, fundus examination showed normal retina and complete veins improvement (Figure 2D).

\section{Histopathological Examination}

The histopathological section for the control retina of albino rabbit showed retinal pigment epithelium (PE), photoreceptor layer $(\mathrm{Ph})$, outer limiting membrane (OLM), outer nuclear layer (ONL), outer plexiform layer (OPL), inner nuclear layer (INL), inner plexiform layer (IPL), ganglion cell layer (GCL), nerve fiber layer (NFL) and inner limiting membrane (ILM), with no significant microscopic alteration in all retina's layers (Figure 3A). Histological examination after one week of rose bengal photodynamic therapy induced BRVO (Figure 3B) showed necrosis and destruction in the surrounding retina, decreasing in thickness and dissolution of the photoreceptor layer with pyknosis of the nuclei of the outer nuclear layer. The inner retinal layers lost its architecture and infiltrated with chronic inflammatory cells. In addition, the capillary of the ganglion cell layer had thick wall and the plexiform layer lost the reticular appearance.

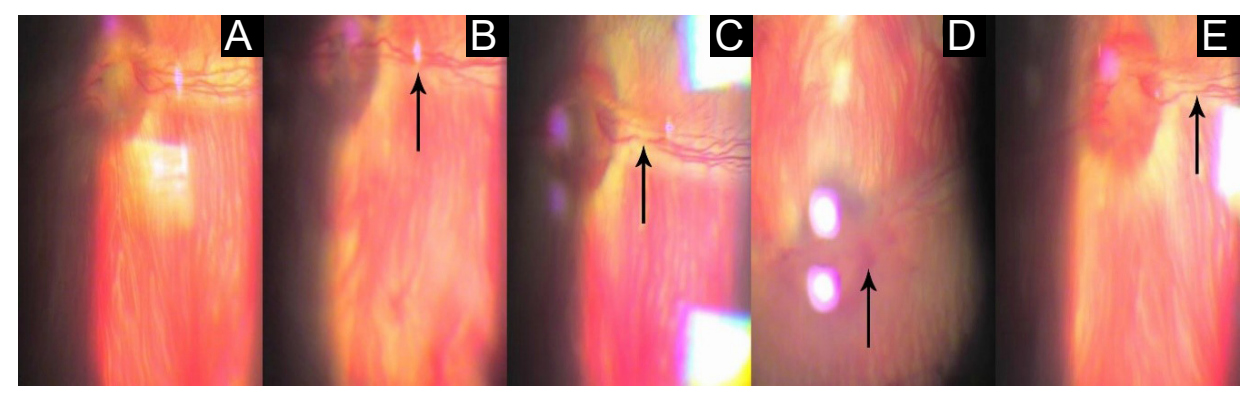

Figure 1. (A) Control rabbit's retina showed normal retina and vascular complex, (B) the location of the applied argon laser, (C) complete branch vein occlusion, (D) hemorrhage around the disc and in the vitreous gel after 4 days of vein occlusion, (E) resolved vitreous and venous thrombosis after 1 week.

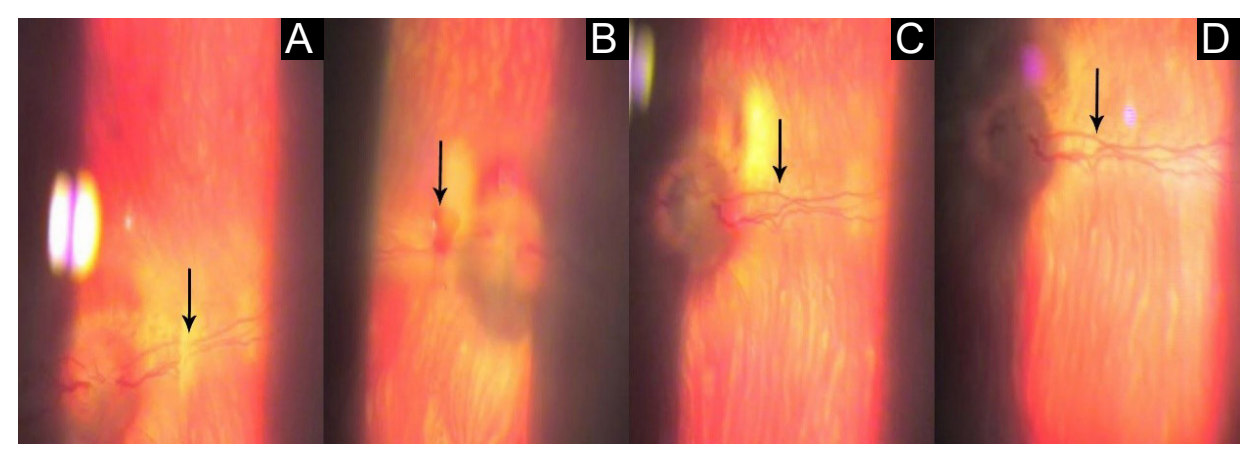

Figure 2. (A) Flow of Blood Stream After Nd: YAG Thrombolysis, (B) Injured Venous Wall With Reflow of Blood Stream, (C) Complete Venous Flow, Minimal Vitreous Hemorrhage and Dry Retina After 1 Week, (D) Normal Retina After 1 Weeks. 


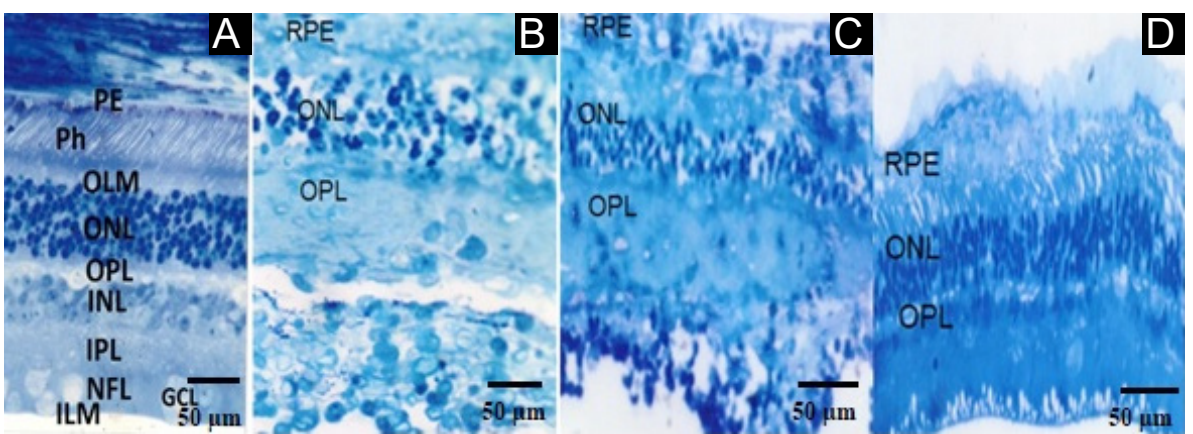

Figure 3. (A) Light micrograph of control rabbit's retina, (B) one week after BRVO by argon laser, the photoreceptor layer was dissolute; there were pyknotic nuclei in the outer nuclear layer. The inner retinal layers lost their architecture and infiltrated with chronic inflammatory cell, (C) One Week After Nd:YAG laser thrombolysis showed slight improvement in all retinal layers, (D) Two weeks after Nd:YAG laser showed nearly improved retinal layers (Toluidine blue, scale bar: $50 \mu \mathrm{m}$ )

All these changes in the retinal layer showed slight improvement after 1 week of treatment with transluminal Nd: YAG laser as shown in Figure 3C especially in the photoreceptor layer, ganglion cells layer and outer nuclear layer. After 2 weeks, the histopathological sections showed more noticeable improvement in the inner segment of photoreceptor layer, the ganglion cell layer (Figure 3D) and the architecture of the inner retina appears nearly normal.

\section{Discussion}

Retinal vein occlusion disease is a common cause of visual loss particularly in the elderly with history of hypertension and arteriosclerotic vascular disease. The occlusion is assumed to be caused by formation of venous thrombosis with different degrees of obstruction to venous outflow. These usually take the form of varying degrees of ischemia, cystoid macular edema, preretinal fibrosis, and pigmentary dispersion. The existing therapeutic options for the treatment of RVO are nonspecific because they fail to report the basic pathological process causing the obstruction in the retinal vein and are limited in their effectiveness. Unfortunately, now there is no means of treatment that is safe and can avoid the progression of a partial retinal vein occlusion to the complete form.

The previous treatments for complete occlusions by laser photocoagulation of the ischemic area aimed to prevent neovascular problems rather than the restoration of vision. ${ }^{27}$ Numerous therapeutic approaches have been tried in the treatment of central retinal venous occlusion, most with limited or no success and were not promising curative treatments to occlusive diseases. Therapies such as x-rays, vitamins, ocular hypotensive agents, anticoagulants and corticosteroids have also proved to be ineffective. ${ }^{28,29}$ Topical pilocarpine and cholesterol lowering agents were used in the past with no scientific basis and were ineffective. ${ }^{30}$ Thrombolytic drugs such as streptokinase have been associated with an unacceptable severe side effects. ${ }^{31}$ In addition, Green et al have recognized that the chronic inflammatory infiltrate found in the area of the thrombosis in the central retinal vein was a consequence of the occlusion rather than the cause. ${ }^{32}$ Current management is depend on some recommendations and new surgical techniques. ${ }^{33,34}$ The applied technique of vitrectomy and artery vein (AV) sheathotomy to separate the artery and vein at the AV crossing induced improvement in visual acuity but it may be accompanied with macular edema, macular hemorrhage, and retinal perfusion. ${ }^{35}$ Restrictions include difficulty in separating the vessels, retinal detachment, hemorrhage and visual field defects. Clot selective agents such as tissue plasminogen activators, offer a theoretical advantage but the results of clinical trials remain to be seen. ${ }^{26}$ Isovolaemic hemodilution has been shown to improve retinal microcirculation with no effect on the extent of obstruction to venous outflow. ${ }^{36}$

More recently, vitrectomy with separation of the posterior hyaloid with or without internal limiting membrane peeling was used for the treatment of macular edema associated with BRVO., ${ }^{37,38}$ This procedure was shown to decrease macular edema and improve visual acuity in some patients. Moreover, treatment of macular edema with intravitreal triamcinolone and focal photocoagulation showed a limited success. ${ }^{39}$ Previously, it was shown that, the vascular endothelial growth factor protein (VEGF) was significantly increased after BRVO. Therefore, the use of anti-VEGF therapy has been introduced for treatment of BRVO-induced macular edema. ${ }^{40}$ None of these previous forms of treatment are widely used due to the lack of effectivity, deleterious side effects and no permanent improvement in the retinal circulation was attained.

In this research, occlusion of the BRV was performed successfully by photodynamic therapy using dye enhanced photothrombosis to induce thrombi in the branch retinal veins. ${ }^{26,41}$ Argon laser causing photosensitization of the rose bengal dye until the blood flow was completely stopped in the vein. Fundus examination revealed vitreous hemorrhage, venous tortuosity, blurred disc edge and hemorrhage around disc with complete vein occlusion. 
The retinal vein obstruction was owing to formation of an intraluminal thrombus. This obstruction blocks venous outflow, producing a complete obstruction and inner retinal hypoxia. Histopathological sections of the retinal layers after one week of photothrombosis revealed changes in the photoreceptor layer, the outer nuclear layer, the inner retinal layers, destruction and necrosis in the surrounding retina (Figure 3B). Additionally, these lesions to retinal layer had similar appearance to those described by Chidlow et al. ${ }^{42}$ Photodynamic therapy would cause collateral lesion via expanding and collapsing vapor bubbles that affected neighboring photoreceptors and the other retinal layer. ${ }^{42}$

The use of thrombolysis for the treatment of BRVO was the subject of intense investigation.

The Nd:YAG laser $(1064 \mathrm{~nm})$, in the infrared range has a precise laser focus spot with small penetrating power causing photodisruption of a thrombus within an occluded BRV. This rapidly increases the temperature of the thrombus between 100 and $305^{\circ} \mathrm{C}$, and partially or completely dislodge the thrombus. ${ }^{43}$ This, in turn, generates both hydrodynamic and acoustic shock waves. The acoustic shock waves are responsible for the desired cleavage of the thrombus, and cavitation bubble formation without harming the venous wall. ${ }^{43}$ Fundus examination showed restoration of retinal blood flow with improvement of the pale colored retina. One week after Nd: YAG laser thrombolysis, histopathological sections, showed slight recovery of retinal layers but rapid perfusion and dramatic improvement of the retina could be accomplished after 2 weeks without collateral damage occurring.

\section{Conclusion}

This research investigates the possibility of creating BRVOs in rabbits' eyes at the site of application of argon laser photothrombosis and targeting the formed thrombus by transluminal Nd: YAG laser thrombolysis as a recent modality in treatment of BRVO with no significant complications. Long term studies are being conducted in order to evaluate the effect of transluminal Nd: YAG laser thrombolysis on the retinal veins and to monitor the possible development of complications.

\section{Ethical Considerations}

All applicable international, national, and / or institutional guide lines for the care and use of animal were followed.

\section{Conflict of Interests}

The authors declare no conflict of interest.

\section{References}

1. Arséne S, Giraudeau B, Le Lez ML, et al. Follow up by colour doppler imaging of 102 patients with retinal vein occlusion over 1 year. Br J Ophthalmol. 2002;86:1243-47.

2. Rogers S, McIntosh RL, Cheung N, et al. The prevalence of retinal vein occlusion:pooled data from population studies from the United States, Europe, Asia, and Australia. Ophthalmology. 2010;117:313-1319. doi:10.1016/j. ophtha.2009.07.017

3. Hamid S, Mirza SA, Shokh I. Branch retinal vein occlusion. J Ayub Med Coll Abbottabad. 2008;20:128-132.

4. Leibreich R. Apoplexia retinae. Graefes Arch Ophthalmol. 1855;1:346-51.

5. Von Michel J. Die spontane thrombose der vena centralis des opticus. Graefes Arch Ophthalmol. 1878;24:37-70.

6. Hayreh SS. Prevalent misconceptions about acute retinal vascular occlusive disorders. Prog Retin Eye Res. 2005;24:493-519. doi:10.1016/j.preteyeres.2004.12.001

7. Rehak M, Wiedemann P. Retinal vein thrombosis: pathogenesis and management. J Thromb Haemost. 2010;8:1886-1894.

8. Duker JS, Brown GC. Anterior location of the crossing artery in branch retinal vein obstruction. Arch Ophthalmol. 1989;107:998-1000.

9. Klein R, Klein BE, Moss SE, Meuer SM. The epidemiology of retinal vein occlusion: The Beaver Dam Eye Study. Trans Am Ophthalmol Soc. 2000;98:133-141.

10. Sperduto RD, Hiller R, Chew E, et al. Risk factors for hemiretinal vein occlusion: comparison with risk factors for central and branch retinal vein occlusion: the eye disease case-control study. Ophthalmology. 1998;105:765771. doi:10.1016/S0161-6420(98)95012-6

11. Recchia FM, Brown GC. Systemic disorders associated with retinal vascular occlusion. Curr Opin Ophthalmol. 2000;11:462-467.

12. Shoch D. Etiologic diagnosis of retinal venous stasis and occlusion. Trans Pac Coast Oto ophthalmol Soc Annu Meet. 1978;51:127.

13. O'Mahoney PRA, Wong DT, Ray JG. Retinal vein occlusion and traditional risk factors for atherosclerosis. Arch Ophthalmol. 2008;126:692-699. doi:10.1001/ archopht.126.5.692

14. Ring CP, Pearson TC, Sanders MD, Wetherley G. Viscosity and retinal vein thrombosis. Br J Ophthalmol. 1976;60:397410.

15. Zhou JQ, Xu L, Wang S, et al. The 10-year incidence and risk factors of retinal vein occlusion: the Beijing eye study. Ophthalmology. 2013;120:803-808. doi:10.1016/j. ophtha.2012.09.033

16. Rehak JR, Rehak $M$ Branch retinal vein occlusion: pathogenesis, visual prognosis, and treatment modalities. Curr Eye Res. 2008;33:111-131. doi:10.1080/02713680701851902

17. Janssen $\mathrm{MCH}$, Heijer M, Cruysberg JRM, et al. Retinal vein occlusion:a form of venous thrombosis or a complication of atherosclerosis? A meta-analysis of thrombophilic factors. Thromb Haemost. 2005;93:1021-1026.

18. Mitchell P, Smith W, Chang A. Prevalence and associations of retinal vein occlusion in Australia: the blue mountains eye study. Arch Ophthalmol. 1996;114:1243-1247.

19. Chatziralli IP, Jaulim A, Peponis VG, et al. Branch retinal vein occlusion: treatment modalities: an update of the literature. Semin Ophthalmol. 2014;29:85-107.

20. Jaulim A, Ahmed B, Khanam T, Chatziralli IP. Branch retinal vein occlusion: epidemiology, pathogenesis, risk factors, clinical features, diagnosis, and complications. An update of the literature. Retina. 2013;33:901-910. 
doi:10.1097/IAE.0b013e3182870c15

21. Jefferies P, Clemett RS, Day T. An anatomical study of retinal arteriovenous crossings and their role in the pathogenesis of retinal branch vein occlusions. Aust NZ J Ophthalmol. 1993;21:213-217.

22. Christoffersen NLB, Larsen M. Pathophysiology and hemodynamics of branch retinal vein occlusion. Ophthalmology. 1999;106:2054-2062. doi:10.1016/S01616420(99)90483-9

23. Zhao J, Sastry SM, Sperduto RD, Chew EY, Remaley NA. Arteriovenous crossing patterns in branch retinal vein occlusion: The Eye Disease Case-Control Study Group. Ophthalmology. 1993;100:423-428.

24. Sekimoto M, Hayasaka S, Setogawa T. Type of arteriovenous crossing at site of branch retinal vein occlusion. Jpn J Ophthalmol. 1992;36:192-196.

25. Spaide RF, Lee JK, Klancnik JK, Gross NE. Optical coherence tomography of branch retinal vein occlusion. Retina. 2003;23:343-347.

26. Oncel M, Peyman GA, Khoobehi B. Tissue plasminogen activator in the treatment of experimental retinal vein occlusion. Retina. 1989;9:1-7.

27. Laatikainen L, Kohner EM, Khoury D, Blach RK. Panretinal photocoagulation in central retinal vein occlusions: A randomised controlled clinical study. $\mathrm{Br} J$ Ophthalmol. 1977;61:741-753.

28. Hesberg RJ. X-ray treatment thrombosis of the retinal vein and of several types of iridocyclitis. Am J Ophthalmol. 1944;27:864-875

29. Vannas S, Orma H. Experience of treating retinal venous occlusion with anticoagulant and antisclerosis therapy. Arch Ophthalmol. 1957;58:812-828.

30. Clements DB, Elsby JM, Smith WD. Retinal vein occlusion: A cooperative study of factors affecting the prognosis, including a therapeutic trial of Atromid-S in this condition. Br J Ophthalmol. 1968;52:111-116.

31. Kohner EM, Pettit JE, Hamilton AM, Bulpitt CJ, Dollery CT. Streptokinase in central retinal vein occlusions: a controlled clinical trial. Br Med J. 1976;1:550-553.

32. Green WR, Chan CC, Hutchins GM, Terry J. Central retinal vein occlusion: a prospective histopathologic study of twenty-nine eyes in twenty-eight cases. Trans Am Soc Ophthalmol. 1981;89:371-422.

33. Branch Vein Occlusion Study Group. Argon laser photocoagulation for macular edema in branch vein occlusion. Am J Ophthalmol. 1984;98:271-282.

34. Branch Vein Occlusion Study Group. Argon laser scatter photocoagulation for prevention of neovascularization and vitreous hemorrhage in branch vein occlusion. A randomized clinical trial. Arch Ophthalmol. 1986;104:3441.

35. Osterloh MD, Charles S Surgical decompression of branch retinal vein occlusions. Arch Ophthalmol. 1988;106:14691471.

36. Hansen LL, Wiek J, Wiederholt M. A randomised prospective study of treatment of non-ischaemic central retinal vein occlusion by isovolaemic haemodilution. $\mathrm{Br} \mathrm{J}$ Ophthalmol. 1989;73:895-899.

37. Saika S, Tanaka T, Miyamoto T, Ohnishi Y. Surgical posterior vitreous detachment combined with gas/air tamponade for treating macular edema associated with branch retinal vein occlusion: retinal tomography and visual outcome. Graefes Arch Clin Exp Ophthalmol. 2001;239:729-732.

38. Mandelcorn MS, Nrusimhadevara RK. Internal limiting membrane peeling for decompression of macular edema in retinal vein occlusion. A report of 14 cases. Retina. 2004;24:348-355.

39. Cekic O, Chang S, Tseng JJ, et al. Intravitreal triamcinolone injection for treatment of macular edema secondary to branch retinal vein occlusion. Retina. 2005;25:851-855.

40. Noma H, Funatsu H, Yamasaki M, et al. Pathogenesis of macular edema with branch retinal vein occlusion and intraocular levels of vascular endothelial growth factor and interleukin-6. Am J Ophthalmol. 2005;140:256-61. doi:10.1016/j.ajo.2005.03.003

41. Genevois O, Paques M, Simonutti M, et al. Microvascular remodeling after occlusion- recanalization of a branch retinal vein in rats. Invest Ophthalmol Vis Sci. 2004;45:594600.

42. Chidlow G, Shibeeb O, Plunkett M, Casson RJ, Wood JP. Glial cell and inflammatory response to retinal laser treatment: comparison of a conventional photocoagulator and a novel, 3-nanosecond pulse laser. Invest Ophthalmol Vis Sci. 2006;54:2319-2332. doi:10.1167/iovs.12-11204

43. Daniel P, Mark B, John W. Retinal laser therapy:Biophysical basis and applications. In: Ryan. SJ, Schachat AP, Wilkinson CP, Hinton DR, Sadda SR, Wiedemann. P, eds. Retina. 5th ed. vol 3. St. Louis: Mosby Inc; 2012. 